\title{
OPINIÃo DE PACIENTES PSIQUIÁTRICOS INTERNADOS SOBRE O HÁBITO DE FUMAR ${ }^{1}$
}

\author{
Edson Arthur Scherer'; Zeyne Alves Pires Scherer'; \\ Hellen Aparecida de Azevedo ${ }^{4}$; Ana Maria Sertori Durão ${ }^{5}$
}

Este é um estudo exploratório-descritivo com abordagem quantitativa e qualitativa, cujo objetivo foi conhecer a opinião dos pacientes acerca do fumar na internação e o grau de dependência dos fumantes. Utilizou-se a entrevista semiestruturada e questionário de Fagerström nos fumantes. Foram observados 25 pacientes: 44,0\% com transtorno de humor, $28,0 \%$ esquizofrenia; $52,0 \% 10$ ou mais anos de doença; $64,0 \% 1$ a 5 internações. Desses, 24,0\% eram fumantes, 83,3\% apresentavam grau elevado de dependência. A maioria dos não fumantes concordou em proibir o fumo em ambientes coletivos, sem privilégios para doentes mentais. Os fumantes mostraram-se contrários a políticas antifumo, mas favoráveis à terapêutica de reposição de nicotina na internação. É esperado o investimento da equipe no ambiente livre de cigarros, nos serviços de saúde e na conscientização do fumante, para buscar tratamento antitabágico.

Descritores: Tabagismo; Pessoas Mentalmente Doentes; Hospitalização; Psiquiatria; Saúde Mental.

\footnotetext{
${ }^{1}$ Apoio financeiro da Fundação de Amparo à Pesquisa do Estado de São Paulo (FAPESP), processo nº $2009 / 05907-9$.

2 Psiquiatra, Doutor em Patologia Experimental, Hospital das Clínicas, Faculdade de Medicina de Ribeirão Preto, Universidade de São Paulo, SP, Brasil.

${ }^{3}$ Enfermeira, Doutor em Enfermagem, Professor Doutor, Escola de Enfermagem de Ribeirão Preto, Universidade de São Paulo, Centro Colaborador da OMS para o Desenvolvimento da Pesquisa em Enfermagem, SP, Brasil. E-mail: scherer@eerp.usp.br.

${ }^{4}$ Aluna do Curso de Graduação em Enfermagem, Escola de Enfermagem de Ribeirão Preto, Universidade de São Paulo, Centro Colaborador da OMS para o Desenvolvimento da Pesquisa em Enfermagem, SP, Brasil. Bolsista de iniciação cientifica do Conselho Nacional de Desenvolvimento Científico e Tecnológico (CNPq). E-mail: li_hellenazevedo@hotmail.com.

${ }^{5}$ Enfermeira, Mestre em Enfermagem, Professor, Centro Universitário Barão de Mauá, Ribeirão Preto, SP, Brasil. Hospital das Clínicas, Faculdade de Medicina de Ribeirão Preto, Universidade de São Paulo, SP, Brasil. E-mail: amsdurão@ig.com.br.
} 


\section{VIEW OF HOSPITALIZED PSYCHIATRIC PATIENTS ON THE SMOKING HABIT}

This is an exploratory descriptive study with quantitative and qualitative approach, which aims to know the opinion of patients about smoking in the hospital and the degree of dependence on smoking. We used a semi-structured interview and Fagerström questionnaire on smokers. We observed 25 patients: $44.0 \%$ with mood disorder, schizophrenia $28.0 \%, 52.0 \% 10$ or more years of disease, $64.0 \% 1-5$ hospitalizations. Of these, $24.0 \%$ were smokers, $83.3 \%$ had a high degree of dependence. Most nonsmokers agreed to ban smoking in collective environments, without privileges for the mentally ill. Smokers were shown to be opposed to smoke-free policies, but in favor of nicotine replacement therapy during hospitalization. It is expected the investment of the team in tobacco-free environment, health services and raise awareness of smokers to seek smoking cessation treatment.

Descriptors: Smoking; Mentally Ill Persons; Hospitalization; Psychiatry; Mental Health.

\section{OPINIÓN DE PACIENTES PSIQUIÁTRICOS INTERNADOS SOBRE EL HÁBITO DE}

\section{FUMAR}

Éste es un estudio exploratorio-descriptivo con abordaje cuantitativo y cualitativo, cuyo objetivo fue conocer la opinión de los pacientes acerca del fumar en la internación y el grado de dependencia de los fumadores. Se utilizó la entrevista semi-estructurada y cuestionario de Fagerström en los fumadores. Fueron observados 25 pacientes: 44,0\% con trastorno de humor, $28,0 \%$ esquizofrenia; $52,0 \% 10$ o más años de enfermedad; $64,0 \% 1$ a 5 internaciones. De eses, 24,0\% eran fumadores, $83,3 \%$ presentaban grado elevado de dependencia. La mayoría de los no fumadores concordó en prohibir el humo en ambientes colectivos, sin privilegios para enfermos mentales. Los fumadores se mostraron contrarios a políticas antitabaquismo, pero favorables a la terapéutica de reposición de nicotina en la internación. Es esperado la inversión del equipo en el ambiente libre de cigarrillos, en los servicios de salud y en la concienciación del fumador, para que busquen tratamiento antitabaquismo.

Descriptores: Tabaquismo; Enfermos Mentales; Hospitalización; Psiquiatría; Salud Mental.

\section{Introdução}

Os doentes mentais constituem um grupo mais vulnerável ao hábito de fumar. O impacto do fumo nessa população foi, por muitos anos, negligenciado, devido a falhas no reconhecimento de que fumar é uma forma de dependência, na lacuna de percepção das complicações médicas desse hábito e no conhecimento limitado sobre a associação entre fumo e distúrbios psiquiátricos ${ }^{(1)}$. Estudos demonstram que a prevalência de tabagismo em pessoas com transtornos psiquiátricos é maior do que na população em geral, inclusive com maiores níveis de tolerância e dependência ${ }^{(1-4)}$. Na situação específica dos hospitais psiquiátricos, resultados de pesquisa realizada em três hospitais de Israel mostraram índices altos de fumantes tanto entre os profissionais de saúde $(48,1 \%)$ quanto entre 
os doentes internados $(76,0 \%)^{(5)}$. Esses autores concluíram que uma pessoa internada nessas circunstâncias é significativamente exposta à "atmosfera pró-fumo" e às consequências do fumo sobre sua saúde.

O tabagismo pode assumir papel significativo na vida dos doentes mentais, pois o consumo de cigarros pode trazer a sensação de alívio dos efeitos colaterais de certas medicações e mesmo de alguns sintomas de doença. O sentimento de desamparo da pessoa portadora de doença mental, aliado à sua desesperança quanto às chances de recuperação e à necessidade de controle, tende a facilitar a busca de alívio no uso do tabaco. A ociosidade, por sua vez, comum nas instituições psiquiátricas, pode contribuir para que o doente fume ${ }^{(3,6)}$.

As dificuldades para o controle do uso do tabaco durante a internação psiquiátrica são devidas, em parte, à não integração de estratégias antifumo às rotinas dos serviços. A descrença dos profissionais de saúde e pacientes que fumam, em relação aos tratamentos para a dependência de nicotina, pode levá-los a interferir em tais abordagens ${ }^{(1)}$. Por outro lado, muitos profissionais da área da saúde acreditam que o doente mental tem o direito de fumar, mesmo em ambientes fechados, como o da internação (questão de direito legal). A liberação do uso do cigarro pode, por sua vez, ser utilizada como forma de garantia para que os pacientes sigam as recomendações do tratamento e participem das diferentes atividades terapêuticas oferecidas. O fumo passa, portanto, a ser visto como instrumento que apazigua e facilita a interação social dos doentes ${ }^{(7)}$. A equipe tende a acreditar que a proibição do uso de cigarros aumentaria a agressividade dos pacientes ${ }^{(4)}$.

Para alguns indivíduos, a internação aparece como forma de diminuir ou mesmo interromper temporariamente o uso do tabaco, o que não é garantia de cessação do seu hábito. Estratégias para cessação do tabagismo que se baseiam em níveis de motivação e preparo para mudar podem, no entanto, não ser apropriadas para doentes mentais $^{(3)}$. Nesse caso, são indicados programas de psicoeducação que ofereçam informações detalhadas acerca da dependência e seus potenciais danos, técnicas e estratégias para controle da ansiedade e manejo do estresse como relaxamento muscular e meditação, associados a intervenções medicamentosas para a abstinência de nicotina, quando indicadas ${ }^{(1,6)}$.

Motivados, portanto, por essas considerações, resolveu-se, aqui, conhecer a opinião dos pacientes acerca do uso de tabaco no ambiente de internação hospitalar e o grau de dependência dos indivíduos fumantes que integram esse grupo.

\section{Método}

Estudo exploratório-descritivo de natureza quantitativa e qualitativa ${ }^{(8)}$, realizado na Enfermaria de Psiquiatria (EPQU) do Hospital das Clínicas da Faculdade de Medicina de Ribeirão Preto da Universidade de São Paulo (HCRP), com 25 pacientes internados.

A EPQU-HCRP tem por objetivo proporcionar internação integral a 15 pacientes psicóticos, em crise aguda, e oferecer rápida reintegração do paciente à sua família e à sociedade. Prioriza o trabalho interdisciplinar realizado por uma equipe fixa (psiquiatra, enfermeiro, auxiliar de enfermagem, psicólogo, assistente social, terapeuta ocupacional e oficial administrativo) e outra flutuante (residentes de psiquiatria, aprimorandos de psicologia, serviço social e terapia ocupacional). Os pacientes participam de um programa de tratamento que consta de farmacoterapia, grupos operativos e de terapia ocupacional, atividades físicas e de lazer, sessões de psicoterapia individual e reuniões de família.

Os pacientes podiam fumar nesse serviço, atendendo algumas determinações relacionadas a local e horário. Desde junho de 2009, foi proibido o fumo nas dependências do HCRP, incluída a EPQU, atendendo a Lei Estadual $\mathrm{n}^{\mathrm{o}} 13.541 / 2009$. Essa Lei proíbe, em todo território do Estado de São Paulo, o uso de cigarros, cigarrilhas, charutos ou qualquer outro produto fumígeno, derivado ou não do tabaco, em ambientes de uso coletivo, público ou privado, total ou parcialmente fechados, compreendendo as instituições de saúde.

$\mathrm{Na}$ EPQU-HCRP, a exemplo do que ocorre no restante da instituição, é oferecido aos indivíduos fumantes internados, como política de restrição ao fumo, o uso de adesivo de nicotina (terapia de reposição de nicotina TRN), mediante sua adesão voluntária. A TRN visa reduzir o desconforto, maximizar a submissão à política antifumo do hospital, e minimizar alguns dos efeitos adrenérgicos indesejáveis associados à abstinência da nicotina ${ }^{(2,9-10)}$. Esse uso segue esquema posológico preconizado para a cessação do hábito de fumar ao longo de sua permanência no serviço.

A coleta de dados foi realizada através da técnica de entrevista semiestruturada. As questões previamente elaboradas continham dados de identificação dos participantes e a opinião desses sobre o hábito de fumar, contemplando os seguintes subitens: reserva de áreas para fumantes em locais públicos, juízo sobre o hábito de fumar entre os doentes mentais, opinião acerca do fumar na EPQU-HCRP, e uma pergunta para identificar em que grupo o entrevistado se enquadra, em relação à utilização ou não do tabaco (nunca fumante, ex-fumante e fumante). Essa questão seleciona os fumantes que responderam o questionário de Fagerström ${ }^{(11)}$, que avalia o grau da dependência do fumante ao tabaco.

Os pacientes internados foram entrevistados na sala de atendimento individual e de grupo da EPQU-HCRP, ao longo de sua permanência na unidade, em horários que não comprometeram sua participação em atividades previstas no seu tratamento.

Para análise dos dados quantitativos, foi construído um banco de dados, utilizando-se o software Excel. Posteriormente, os mesmos foram compilados através do software SPSS. Os dados qualitativos foram submetidos à técnica de análise de conteúdo temática ${ }^{(8)}$.

A pesquisa foi aprovada pelo comitê de ética do HCRP (Processo HCRP n³504/2009), atendendo às orientações inerentes ao protocolo de pesquisa contido na Resolução 196/96, CNS. 


\section{Resultados}

Os resultados referentes às características sociodemográficas dos pacientes estudados são apresentados na Tabela 1 .

Tabela 1 - Características sociodemográficas da população do estudo. Ribeirão Preto, SP, Brasil, 2010

\begin{tabular}{|c|c|c|}
\hline \multirow{2}{*}{ Variáveis } & \multicolumn{2}{|c|}{ Pacientes } \\
\hline & f & $\%$ \\
\hline \multicolumn{3}{|l|}{ Idade } \\
\hline $12 \vdash 20$ & 5 & 20,0 \\
\hline $20 \vdash 30$ & 1 & 4,0 \\
\hline $30 \vdash 40$ & 7 & 28,0 \\
\hline $40 \vdash 50$ & 2 & 8,0 \\
\hline $50 \vdash 60$ & 5 & 20,0 \\
\hline 60 ou mais & 5 & 20,0 \\
\hline \multicolumn{3}{|l|}{ Sexo } \\
\hline Masculino & 12 & 48,0 \\
\hline Feminino & 13 & 52,0 \\
\hline \multicolumn{3}{|l|}{ Nupcialidade } \\
\hline Casado & 7 & 28,0 \\
\hline Solteiro & 13 & 52,0 \\
\hline União consensual & 1 & 4,0 \\
\hline Separado & 2 & 8,0 \\
\hline Viúvo & 2 & 8,0 \\
\hline \multicolumn{3}{|l|}{ Escolaridade } \\
\hline Fundamental incompleto & 8 & 32,0 \\
\hline Fundamental completo & 3 & 12,0 \\
\hline Médio incompleto & 2 & 8,0 \\
\hline Médio completo & 7 & 28,0 \\
\hline Superior incompleto & 3 & 12,0 \\
\hline Superior completo & 2 & 8,0 \\
\hline \multicolumn{3}{|l|}{ Religião } \\
\hline Sem religião & 3 & 12,0 \\
\hline Católico & 15 & 60,0 \\
\hline Pentecostal & 4 & 16,0 \\
\hline Protestante & 1 & 4,0 \\
\hline Espírita & 2 & 8,0 \\
\hline \multicolumn{3}{|l|}{ Ocupação* } \\
\hline Grupo 2 & 1 & 4,0 \\
\hline Grupo 3 & 2 & 8,0 \\
\hline Grupo 5 & 12 & 48,0 \\
\hline Grupo 7 & 3 & 12,0 \\
\hline Grupo 9 & 1 & 4,0 \\
\hline Aposentado & 1 & 4,0 \\
\hline Estudante & 4 & 16,0 \\
\hline Inativo & 1 & 4,0 \\
\hline
\end{tabular}

*Ocupação/profissão conforme Grandes Grupos Ocupacionais da Classificação Brasileira de Ocupações (CBO - Ministério do Trabalho e Emprego): Grupo 2 - profissionais das ciências e das artes; Grupo 3 - técnicos de nível médio; Grupo 5 - trabalhadores dos serviços, vendedores do comércio em lojas e mercados; Grupo 7 - trabalhadores da produção de bens e serviços industriais; Grupo 9 - trabalhadores de manutenção e reparação.

Foram entrevistados 25 pacientes, sendo que 12 (48\%) eram do sexo masculino. A maioria era adulta, na faixa dos 20 aos 59 anos (60,0\%). Quanto à nupcialidade, $17(68,0 \%)$ não tinham vínculo nupcial (solteiros, viúvos ou separados) e $8(32,0 \%)$ tinham (casados ou com união consensual). Do total de 25 pacientes, $8(32,0 \%)$ tinham ensino fundamental incompleto; 5 (20,0\%) ensino fundamental completo ou médio incompleto; 10 (40,0\%) declararam ter ensino médio completo ou superior incompleto e $2(8,0 \%)$ ensino superior completo. No que diz respeito às ocupações, $12(48,0 \%)$ dos pacientes exerciam atividades ocupacionais relacionadas ao grupo de trabalhadores dos serviços (dona de casa, faxineira, vendedor, balconista, serviços gerais); 3 (12,0\%) aos de produção de bens e serviços industriais (motorista, pedreiro, comerciante de equipamentos industriais); 2 $(8,0 \%)$ aos técnicos de nível médio (bancário, auxiliar de enfermagem); 1 (4,0\%) aos profissionais das ciências e das artes (jornalista); $1(4,0 \%)$ aos operários de manutenção (rasteleiro de asfalto); 1 (4,0\%) declarou estar inativo; $1(4,0 \%)$ aposentado e $4(16,0 \%)$ eram estudantes. Dos 25 pacientes, $15(60,0 \%)$ eram católicos, $4(16,0 \%)$ pentecostais, $1(4,0 \%)$ protestante, $2(8,0 \%)$ espíritas e 3 $(12,0 \%)$ sem religião.

Apresentam-se, na Tabela 2, informações das características clínicas relativas aos doentes, compreendendo o grupo diagnóstico, duração da doença e da internação atual, número de internações, uso de tabaco e grau de dependência dos fumantes (avaliado pelo questionário de Fagerström).

Tabela 2 - Diagnóstico, duração da doença e da internação atual, número de internações, uso de tabaco dos pacientes do estudo e grau de dependência dos fumantes. Ribeirão Preto, SP, Brasil, 2010

\begin{tabular}{|c|c|c|}
\hline \multirow{2}{*}{ Características } & \multicolumn{2}{|c|}{ Pacientes } \\
\hline & f & $\%$ \\
\hline \multicolumn{3}{|l|}{ Grupo diagnóstico - CID-10 } \\
\hline F00 - F09 & 1 & 4,0 \\
\hline $\mathrm{F} 10-\mathrm{F} 19$ & 1 & 4,0 \\
\hline $\mathrm{F} 20-\mathrm{F} 29$ & 7 & 28,0 \\
\hline$F 30-F 39$ & 11 & 44,0 \\
\hline$F 40-F 48$ & 1 & 4,0 \\
\hline F50 - F59 & 1 & 4,0 \\
\hline F60 - F69 & 3 & 12,0 \\
\hline \multicolumn{3}{|l|}{ Tempo de doença (anos) } \\
\hline $0 \vdash 1$ & 4 & 16,0 \\
\hline $1 \vdash 5$ & 6 & 24,0 \\
\hline $5 \vdash 10$ & 2 & 8,0 \\
\hline $10 \vdash 20$ & 10 & 40,0 \\
\hline 20 ou mais & 3 & 12,0 \\
\hline \multicolumn{3}{|l|}{ Tempo internação atual (dias) } \\
\hline $0+30$ & 14 & 56,0 \\
\hline $30 \vdash 60$ & 5 & 20,0 \\
\hline $60 \vdash 90$ & 2 & 8,0 \\
\hline $90 \vdash-120$ & 3 & 12,0 \\
\hline 120 ou mais & 1 & 4,0 \\
\hline \multicolumn{3}{|l|}{ Número de internações } \\
\hline 1 & 8 & 32,0 \\
\hline 2 a 5 & 8 & 32,0 \\
\hline
\end{tabular}


Tabela 1 - continuação

\begin{tabular}{lcc}
\hline \multirow{2}{*}{ Características } & \multicolumn{2}{c}{ Pacientes } \\
\cline { 2 - 3 } & f & $\%$ \\
\hline 6 ou mais & 9 & 36,0 \\
Uso do tabaco & & \\
Fumante & 6 & 24,0 \\
Nunca fumante & 17 & 68,0 \\
Ex-fumante & 2 & 8,0 \\
Grau de dependência ${ }^{\dagger}$ & & \\
Muito baixo & 0 & 00,0 \\
Baixo & 1 & 16,7 \\
Médio & 0 & 00,0 \\
Elevado & 4 & 66,6 \\
Muito elevado & 1 & 16,7 \\
\hline
\end{tabular}

†Grupo diagnóstico principal da CID-10 que motivou a internação do paciente. Nomenclatura: F00 - F09 transtornos mentais orgânicos, inclusive os sintomáticos; F10-F19 transtornos mentais e comportamentais devidos ao uso de substância psicoativa; F20 - F29 esquizofrenia, transtornos esquizotípicos e transtornos delirantes; F30 - F39 transtornos do humor; F40 - F48 transtornos neuróticos, transtornos relacionados com o stress e transtornos somatoformes; F50 - F59 síndromes comportamentais associadas a disfunções fisiológicas e a fatores físicos; F60 - F69 transtornos da personalidade e do comportamento do adulto.

"Resultados da aplicação do questionário de Fagerström nos indivíduos que se declararam fumantes.

Neste estudo, aproximadamente três quartos dos pacientes internados receberam diagnóstico de transtorno de humor $(44,0 \%)$ ou de esquizofrenia $(28,0 \%)$. Também foi observada menor proporção de transtornos relacionados ao uso de substâncias psicoativas (4,0\% x 7,3\%) e transtornos psicorgânicos $(4,0 \% \times 9,3 \%)$. Dos pacientes que participaram do estudo, $13(52,0 \%)$ tinham história de 10 ou mais anos de evolução de doença, 8 (32,0\%) referiram de 1 a 9 anos de evolução e 4 (16,0\%) menos de um ano desde o início de seu padecimento. Quanto ao número de internações, $16(64,0 \%)$ tinham história de 1 a 5 internações e $9(36,0 \%)$ de 6 ou mais. Em relação à duração da internação atual, a média foi de 46,3 dias, mediana de 30 dias, com variação de 3 a 240 dias.

Do total de 25 pacientes que responderam a presente pesquisa, $17(68,0 \%)$ nunca fumaram, ou seja, fumaram menos de 5 maços de cigarros (ou menos de 100 cigarros) durante toda a vida; $2(8,0 \%)$ eram ex-fumantes, portanto, fumaram 5 maços ou mais de cigarros ( 100 ou mais cigarros) durante toda a vida e, atualmente, não fumam mais. Os $6(24,0 \%)$ restantes eram fumantes, caracterizados como aqueles que fumaram 5 maços de cigarros ou mais (100 ou mais cigarros) durante toda a vida, e fumam atualmente cigarros ou derivados (cachimbos, charuto, rapé ou tabaco mascado).

Na Tabela 3, estão apresentados os resultados acerca da opinião dos participantes do estudo sobre a reserva de áreas para fumar e sua proibição.

$\mathrm{Na}$ opinião da maioria dos entrevistados $(92,0 \%)$, deveriam ser reservadas áreas específicas para os fumantes e ser proibido o uso de tabaco em locais onde costuma haver concentração de pessoas. Vinte e três pacientes (92,0\%) concordam com a proibição do uso de cigarros em aeroportos, igrejas, transportes coletivos, hospitais e $21(84,0 \%)$ em centros comerciais. Todos os pacientes fumantes manifestaram ser favoráveis à reserva de áreas para fumantes em locais públicos. Deve ter uma área para fumantes (P5). Não deve fumar em lugar proibido, deve ir para outro lugar (P8). Tendo um lugar reservado é melhor (P19).

Tabela 3 - Opinião dos pacientes sobre reserva de áreas para fumar e sua proibição. Ribeirão Preto, SP, Brasil, 2010

\begin{tabular}{lcc}
\hline \multirow{2}{*}{ Variáveis } & \multicolumn{2}{c}{ Pacientes } \\
\cline { 2 - 3 } & $\mathbf{f}$ & $\%$ \\
\hline Reserva de áreas para fumar & 2 & 8,0 \\
$\quad$ Não & 23 & 92,0 \\
Sim & & \\
Proibir fumo em aeroporto & 2 & 8,0 \\
$\quad$ Não & 23 & 92,0 \\
Sim & & \\
Proibir fumo em igrejas & 2 & 8,0 \\
$\quad$ Não & 23 & 92,0 \\
$\quad$ Sim & & \\
Proibir fumo em transportes coletivos & 2 & 8,0 \\
$\quad$ Não & 23 & 92,0 \\
Sim & & \\
Proibir fumo em centros comerciais & \multicolumn{2}{c}{16,0} \\
$\quad$ Não & 4 & 16,0 \\
Sim & 21 & 84,0 \\
Proibir fumo em hospitais & & \\
$\quad$ Não & 2 & 8,0 \\
Sim & 23 & 92,0 \\
\hline
\end{tabular}

A Tabela 4 mostra os achados acerca da opinião dos sujeitos entrevistados sobre o uso de cigarros, pelos doentes mentais, em diferentes locais.

Tabela 4 - Opinião dos sujeitos entrevistados sobre o uso de cigarros pelos doentes mentais em diferentes locais. Ribeirão Preto, SP, Brasil, 2010

\begin{tabular}{|c|c|c|}
\hline \multirow{2}{*}{ Variáveis } & \multicolumn{2}{|c|}{ Pacientes } \\
\hline & f & $\%$ \\
\hline \multicolumn{3}{|c|}{$\begin{array}{l}\text { Permitir a doente mental fumar em local público onde é } \\
\text { proibido }\end{array}$} \\
\hline Não & 22 & 88,0 \\
\hline Sim & 2 & 8,0 \\
\hline Não sei & 1 & 4,0 \\
\hline \multicolumn{3}{|c|}{ Permitir a doente mental fumar em HD§ } \\
\hline Não & 15 & 60,0 \\
\hline Sim & 9 & 36,0 \\
\hline Não sei & 1 & 4,0 \\
\hline \multicolumn{3}{|c|}{ Permitir a doente mental fumar em ambulatórios } \\
\hline Não & 22 & 88,0 \\
\hline Sim & 3 & 12,0 \\
\hline \multicolumn{3}{|c|}{ Permitir a doente mental fumar em CAPS2 } \\
\hline Não & 15 & 60,0 \\
\hline Sim & 9 & 36,0 \\
\hline Não sei & 1 & 4,0 \\
\hline
\end{tabular}

(continua...) 
Tabela 4 - continuação

\begin{tabular}{lcc}
\hline \multicolumn{1}{c}{ Variáveis } & \multicolumn{2}{c}{ Pacientes } \\
\cline { 2 - 4 } & $\mathbf{f}$ & $\%$ \\
\hline $\begin{array}{l}\text { Permitir a doente mental fumar em enfermarias de } \\
\text { hospital geral }\end{array}$ & & \\
$\quad$ Não & 19 & 76,0 \\
Sim & 6 & 24,0 \\
Sente dificuldade ou incômodo com fumo na EPQU3 & & \\
Não & 9 & 36,0 \\
Sim & 16 & 64,0 \\
Proibir fumo na EPQU & & \\
Não & 8 & 32,0 \\
Sim & 17 & 68,0 \\
Substituir fumo por adesivo de nicotina na EPQU & \multicolumn{2}{c}{} \\
$\quad$ Não & 2 & 8,0 \\
Sim & 22 & 88,0 \\
Não sei & 1 & 4,0 \\
\hline
\end{tabular}

${ }^{8} \mathrm{HD}$ - hospitais-dia; ${ }^{2} \mathrm{CAPS}$ - Centros de Atenção Psicossocial.; ${ }^{3}$ os pacientes podiam fumar na EPQU até junho de 2009, quando foi proibido o fumo em todo o HCRP.

Os pacientes concordam, em número de $22(88,0 \%)$, dentre os quais 4 fumantes $(66,7 \%$ desses), com a não permissão a doentes mentais para utilizarem o tabaco em locais públicos, onde é proibido fumar. Entre as justificativas, mencionam os malefícios do cigarro, o prejuízo para o tratamento e para as pessoas que não são fumantes. Se é proibido é porque faz mal, senão, não seria proibido (P2). Faz parte do tratamento você ficar sem o cigarro. Cigarro é um grande maleficio para o cérebro (P25). Mesmo que tenha problema mental, atrapalha a saúde dos doentes (P7). Porque é melhor para o tratamento da pessoa e para quem não fuma (P8).

Os $2(33,3 \%)$ fumantes que se mostraram favoráveis a que os doentes mentais fumem em locais públicos, onde é proibido, usaram como argumento o direito do cidadão ao livre-arbítrio e a noção de que o cigarro alivia a ansiedade. Cada um faz o que quer (P6). O cigarro alivia a ansiedade (P21).

Perguntados sobre a liberação do fumo para doentes mentais em serviços especializados de atendimento, a maioria dos entrevistados é contra o uso em ambulatórios $(88,0 \%)$, mesmo entre os fumantes $(83,3 \%)$. Em relação às EPQUs, a maioria dos pesquisados concorda, também, em não permitir o uso de cigarros pelos internados $(76,0 \%)$. Porque prejudica quem não fuma, o não fumante recebe a maior carga (P12). Proibindo enquanto eles estão aqui, eles podem pensar em parar quando estiverem lá fora (P24).

Ao se questionar, no entanto, acerca da proibição do uso do tabaco na EPQU-HCRP, apesar de a maioria dos sujeitos concordarem, os achados foram em percentual menor $(68,0 \%)$. Já o grupo dos fumantes mostrou-se dividido: $3(50,0 \%)$ favoráveis e $3(50,0 \%)$ contrários à proibição do tabaco em EPQUs e, em sua maioria (66,6\%), contrários a proibir o uso de cigarros pelos pacientes durante internação na EPQU-HCRP. O esperado seria que as respostas a essas duas questões fossem semelhantes. Deve ter uma área para fumante (P5). Porque não vou lá quando estão fumando (P1). Tolero para não ser antissocial (P10).

Quando questionados se encontravam alguma dificuldade ou sentiam algum incômodo decorrente do uso de cigarros pelos internos na EPQU-HCRP, 64,0\% dos pacientes responderam afirmativamente. Todos os sujeitos fumantes responderam negativamente a essa questão. $O$ cheiro incomoda as pessoas (P1). Sinto enjoo e dor de cabeça (P8). Porque é muito ruim, porque o cheiro é ruim, incomoda (P9). Não, porque também fumo e estou acostumada com o cheiro (P21).

Os sujeitos pesquisados, em sua maioria $(88,0 \%)$, concordam com a conduta de oferecer o uso de adesivo de nicotina (TRN) aos indivíduos fumantes internados na EPQU-HCRP, como política de restrição ao fumo. Dos fumantes, $4(66,6 \%)$ se posicionaram favoravelmente ao TRN. A pessoa tem que parar de uma vez (P4). Porque já estou usando, é proveitoso, não estou sentindo vontade de fumar (P7). Diminui a dependência aos poucos, se houver abstinência rigorosa, a pessoa fuma dobrado (P10). Assim, a pessoa não lembra do cigarro (P18).

\section{Discussão}

O Ministério da Saúde realizou, entre 2002 e 2003, o "Inquérito domiciliar sobre comportamentos de risco e morbidade referida de doenças e agravos não transmissíveis", em 15 capitais brasileiras e no Distrito Federal. Nesse inquérito, foi mostrado que a prevalência total de tabagismo na população acima de 15 anos foi de cerca de $19 \%$, com incidência maior entre os homens ${ }^{(12-13)}$. Achados mais recentes do "Sistema de Vigilância de Fatores de Risco e Proteção para Doenças Crônicas não Transmissíveis por Inquérito Telefônico - VIGITEL", implantado pelo Ministério da Saúde, apontam, em 2006, ano de seu primeiro levantamento, percentual de 16,2\% de tabagistas no Brasil, hábito mais disseminado entre homens $^{(14)}$.

Neste estudo, o percentual de fumantes foi de $24,0 \%$, resultado superior ao dos estudos populacionais. A prevalência de fumo entre pacientes em hospitais tende a ser maior ${ }^{(15)}$. Pesquisa realizada em hospital geral universitário de São Paulo, no ano 1995, identificou $21 \%$ de pacientes fumantes em diferentes unidades especializadas de internação dessa instituição ${ }^{(16)}$. Pacientes internados em uma enfermaria de psiquiatria de um hospital universitário de Porto Alegre foram submetidos a três censos consecutivos, em intervalo que atendeu a rotatividade das internações, tendo sido encontrados os percentuais de 46,7, 54,3 e 38,9\% de doentes fumantes em cada momento $^{(17)}$. Quanto ao sexo, 53,3\% dos tabagistas eram mulheres, na ocasião do primeiro censo; no segundo, $57,9 \%$ eram homens; e, no terceiro, a proporção entre homens e mulheres foi idêntica. Os resultados aqui apresentados, quanto ao número total de pacientes tabagistas, ficaram mais próximos de estudo realizado no hospital de São Paulo (21\%), com percentual de 24,0\% de doentes internados fumantes. Quanto à prevalência do tabagismo no que se refere ao sexo, no total foram 4 $(66,6 \%)$ mulheres e $2(33,3 \%)$ homens fumantes, achado diferente do encontrado nos estudos populacionais.

Ao se comparar a distribuição diagnóstica deste 
estudo com dados de outra pesquisa ${ }^{(18)}$, encontrou-se maior proporção de transtorno de humor (neste trabalho: 44,0\%; dado da outra pesquisa: $30,0 \%$ ) e de esquizofrenia (aqui: 28,0\%; dado da outra pesquisa: $19,1 \%$ ). Em relação ao diagnóstico de transtornos neuróticos ou de personalidade, os resultados foram similares (este estudo: 16,0\%; dado da outra pesquisa: $15,0 \%$ ). Houve, ainda, nestes achados, 1 (4\%) paciente com diagnóstico de síndrome comportamental associada a disfunções fisiológicas e a fatores físicos.

O grau de dependência, avaliado pela aplicação do questionário de Fagerström, foi considerado elevado (escore $\geq 6$ ) para $5(83,3 \%)$ dos pacientes fumantes. Esse achado corrobora dados da literatura que sugerem que pacientes psiquiátricos apresentam dependência mais intensa do que indivíduos que fumam e não apresentam diagnóstico de doença mental comórbida ${ }^{(17)}$.

Em relação ao uso de tabaco em locais públicos, ou seja, onde costuma haver concentração de pessoas, $92,0 \%$ dos entrevistados concordam em proibir o fumo. Esses achados foram semelhantes aos da Itália e Irlanda, onde, respectivamente, 90,0 e $83,0 \%$ da população se manifestaram a favor de áreas públicas livres de tabaco ${ }^{(19)}$.

Os indivíduos fumantes, em geral, tendem a não concordar com normas que interfiram em seu direito de fumar em locais públicos fechados, como hospitais e outros serviços de assistência à saúde ${ }^{(20)}$. Da mesma forma, neste estudo, todos os tabagistas foram favoráveis à reserva de áreas para fumantes em locais públicos.

De acordo com a literatura ${ }^{(21)}$, o uso do tabaco causa prejuízo à integridade física e psicológica do indivíduo. A nicotina é indutora da dependência, e fatores individuais, sociais e ambientais são importantes para o desenvolvimento dessa adição ${ }^{(21)}$.Nesta pesquisa, a maioria dos pacientes (22-88,0\%) concordou em não permitir que doentes mentais utilizem o tabaco em locais públicos, onde é proibido. Apresentaram como justificativa para essa postura o seu conhecimento acerca dos malefícios do cigarro, do prejuízo para o tratamento e para as pessoas que não são fumantes.

O hábito de fumar pode funcionar como um tampão emocional, pois o fumante tende a acreditar que, ao fumar, consegue controlar sua ansiedade, ira, tristeza, ficando mais calmo ${ }^{(6,21)}$. No presente estudo, aqueles fumantes favoráveis a que os doentes mentais fumem em locais públicos, onde é proibido, usaram como argumento o direito de cada cidadão ao livre-arbítrio e a noção de que o cigarro alivia a ansiedade. O fumo contribui, no entanto, para o desenvolvimento de distúrbios de ansiedade (agorafobia, pânico e ansiedade generalizada) ${ }^{(22)}$. Isso seria relacionado, entre outros, aos presumíveis efeitos ansiogênicos da nicotina. Por outro lado, a cessação do fumo é seguida pela redução da ansiedade, após 4 semanas de abstinência ${ }^{(22)}$. A questão que se refere ao direito de fumar (livre-arbítrio) não será abordada neste estudo, por ser tema polêmico e fugir dos objetivos propostos.

A questão referente à liberação do fumo para doentes mentais, em serviços de atendimento de saúde mental, mostrou resultados divergentes. Apesar de a maioria dos não fumantes concordar com a não permissão do uso de cigarros pelos pacientes em HD e CAPS, os percentuais foram menores em relação à sua proibição nos ambulatórios. Essa diferença pode ser explicada pelo fato de que os pacientes, em geral, permanecem por período de tempo maior em HD e CAPS do que em ambulatórios. Nesses, os doentes comparecem para o atendimento e, ao término do mesmo, retornam para seus domicílios. Outra possível justificativa pode ser relativa ao espaço físico maior dos HDs e CAPS que, por sua vez, permitiria reservar áreas para fumantes.

Os fumantes deste estudo, em sua maioria, permitiriam o fumo em HD e CAPS. Além da justificativa acima apresentada, outra motivação pode ser a encontrada em estudo ${ }^{(23)}$ conduzido em um CAPSad (CAPS álcool e drogas), no qual os pacientes, antes de frequentarem o tratamento, não consideravam o cigarro como uma droga ilícita. Acreditavam que poderiam fumar livremente e que o cigarro não causa danos à saúde.

$\mathrm{Na}$ situação específica da permissão de fumar em internações hospitalares, em EPQUs, a maioria dos sujeitos não fumantes deste estudo concordou com a proibição do fumo. Os fumantes, por sua vez, mostraram-se divididos, metade concordando com essa proibição e metade discordando. Quando questionados especificamente acerca da EPQU-HCRP, houve queda no número global de respostas favoráveis à proibição do fumo. A maioria dos não fumantes e apenas um terço dos fumantes concordaram com a proibição do uso do cigarro durante a internação na EPQU-HCRP. Como esclarecido anteriormente, os pacientes podiam fumar na EPQU-HCRP até junho de 2009, quando foi determinada sua proibição. Portanto, os doentes já estavam acostumados com uma enfermaria onde era permitido o uso de cigarros, ou seja, havia certa "cultura pró-fumo dos pacientes"(5). Isso poderia explicar as diferenças nas respostas desses grupos quanto à permissão do fumo em EPQUs e no serviço onde esse estudo foi realizado.

A maioria dos não fumantes referiu sentir dificuldades ou incômodos em ambientes de internação onde é permitido fumar. Houve manifestações de inconformismo com o fato de serem expostos ao fumo passivo, de esforço para tolerar o convívio em tais ambientes, incômodo com o cheiro do cigarro, queixas quanto a sentirem enjoo e dor de cabeça. Já os fumantes, pelo próprio hábito, negaram qualquer dificuldade ou incômodo e sugeriram a reserva de área nas enfermarias para fumantes. Os não fumantes, por outro lado, manifestaram a ideia de que a abstinência do cigarro na internação poderia servir de estímulo para a cessação do hábito após a alta hospitalar.

Apesar das diferenças encontradas entre os fumantes e os não fumantes, a maioria dos sujeitos dos dois grupos concordou com a conduta atual da EPQU-HCRP do uso de adesivo de nicotina como TRN, garantindo atmosfera livre de fumo no serviço. 


\section{Considerações Finais}

Conhecer a opinião das pessoas que ficarão sob o cuidado de uma equipe de saúde, em especial em situação de internação hospitalar, facilita a abordagem de situações que podem gerar dúvidas ou mesmo conflitos, como é a referente à restrição ou proibição de uso de alguma substância pelo indivíduo. Neste estudo, foi possível perceber diferenças de opinião de pacientes fumantes e não fumantes sobre proibir os doentes mentais de fumarem em serviços de atendimento psiquiátrico. Apesar dessas diferenças, houve concordância dos entrevistados em relação ao uso voluntário da TRN pelos indivíduos fumantes ao longo de sua internação na EPQU-HCRP, atendendo ao preconizado pela lei estadual que proibiu o uso de cigarros em ambientes fechados.

A equipe de assistência tem papel ativo na inclusão dos doentes nos diferentes serviços disponíveis na rede de atenção à saúde. Nesse sentido, precisa oferecer condições para que os pacientes convivam uns com os outros e com os profissionais, em ambiente de harmonia. Trabalhar o comportamento em relação ao uso do tabaco junto aos fumantes e não fumantes implica melhorar sua interação e ajudá-los a adotar estilo de vida saudável, valorizando os fatores protetores da saúde individual e coletiva. A equipe de assistência precisa estar atenta ao fato de que oferecer ambiente livre de fumo nos serviços de saúde pode constituir o início de um processo terapêutico para estimular a cessação do hábito de fumar. É esperado, portanto, que os profissionais abordem a questão do fumo em sua totalidade, ou seja, não apenas no tocante à proibição do mesmo, e à introdução de TRN nos serviços de tratamento, mas investindo na prevenção e conscientização do fumante para buscar tratamento antitabagismo.

\section{Referencias}

1. Rosen-Chase C, Dyson V. Treatment of Nicotine Dependence in the Chronic Mentally Ill. J Substance Abuse Treat. 1999;16(4):315-20.

2. Prochaska JJ, Gill P, Hall SM. Treatment of Tobacco Use in an Inpatient Psychiatric Setting. Psychiatr Serv. 2004;55:1265-70.

3. Lawn S, Pols R. Smoking bans in psychiatric inpatient settings? A review of the research. Austr N Z J Psychiatry. 2005;39:866-85.

4. Reilly P, Murphy L, Alderton D. Challenging the smoking culture within a mental health service supportively. Int J Mental Health Nurs. 2006;15:272-8.

5. Mester R, Toren P, Ben-Moshe Y, Weyzeman A. Suvery of smoking habits and attitudes of patients and staff in psychiatric hospitals. Psychopathology. 1993;26(2):69-75. 6. Rondina RC, Goyareb R, Botelho C. Características psicológicas associadas ao comportamento de fumar tabaco. J Bras Pneumol. 2007;33(5):592-601.
7. Lawn S. Cigarette smoking in psychiatric settings: occupational health, safety, welfare and legal concerns. Austr N Z J Psychiatry. 2005;39:886-91.

8. Minayo MCS. O desafio do conhecimento: pesquisa qualitativa em saúde. São Paulo/Rio de Janeiro: HUCITEC/ ABRASCO; 1992.

9. Laranjeira R, Giglioti A. Tratamento da dependência de nicotina. Psiq Prat Med. 2000;33(2):9-18.

10. Focchi Gr, Braun Im. Tratamento farmacológico do tabagismo. Rev Psiquiatr Clín. 2005;32(5):259-66.

11. Meneses-Gaya IC, Zuardi AW, Loureiro SR, Crippa JAS. As propriedades psicométricas do Teste de Fagerström para Dependência de Nicotina. J Bras Pneumol. 2009;35(1):73-82.

12. Cavalcante TM. O controle do tabagismo no Brasil: avanços e desafios. Rev Ppsiquiatr Clín. 2005;32(5):283300 .

13. Corrêa PCRP, Barreto SM, Passos VMA. Métodos de estimativa da mortalidade atribuível ao tabagismo: uma revisão literária. Epidemiol Serv Saúde. 2008;17(1):43-57. 14. Moura EC, Morais OL Neto, Malta DC, Moura L, Silva NN, Bernal R, et al. Vigilância de Fatores de Risco para Doenças Crônicas por Inquérito telefônico nas capitais dos 26 estados brasileiros e no Distrito Federal (2006). Rev Bras Epidemiol. 2008;11(supl 1):20-37.

15. Willaing I, Jørgensen T, Iversen L. How does individual smoking behaviour among hospital staff influence their knowledge of the health consequences of smoking? Scand J Public Health. 2003;31:149-55.

16. Figlie NB, Pillon SC, Dunn J, Laranjeira R. The frequency of smoking and problem drinking among general hospital inpatients in Brazil - using the AUDIT and Fagerström questionnaires. São Paulo Med J. 2000;118(5):139-43.

17. De Boni R, Pechansky F. Prevalência de tabagismo em uma unidade de internação psiquiátrica de Porto Alegre. Rev Psiquiatr. RS. 2003;25(3):475-8.

18. Dalgalarrondo P, Botega NJ, Banzato CEM. Pacientes que se beneficiam de internação psiquiátrica em hospital geral. Rev Saúde Pública. 2003;37(5):629-34.

19. Lemstra M, Neudorf C, Oponda J. Implications of a public smoking ban. Can J Public Health. 2008;99(1):625.

20. Gokirmak M, Ozturk O, Bircan A, Akkaya A. The attitude toward tobacco dependence and barriers to discussing smoking cessation: a survey among Turkish general practitioners. Int J Public Health. 2010;55:177-83. 21. Consuegra RVG, Zago MMF. Creencias en fumadores pertenecientes a un programa de salud cardiovascular. Rev. Latino-Am. Enfermagem. 2004;12(nº esp):412-9.

22. Johnson JG, Cohen P, Pine DS, Klein DF, Kasen S, Brook JS. Association Between Cigarette Smoking and Anxiety Disorders During Adolescence and Early Adulthood. JAMA. 2000;284:2348-51. 
23.Vieira JKS, Carvalho RN, Azevedo EB, Silva PMC, Ferreira MO Filha. Concepção sobre drogas: Relatos dos usuários do CAPS-ad, de Campina Grande, PB. SMAD, Rev. Eletrônica Saúde Mental Álcool Drog. (Ed. port.) [periódico na Internet]. 2010 [acesso 4 fev 2010];6(2):27495. Disponível em: http://www2.eerp.usp.br/resmad/ artigos.php $?$ idioma $=$ portugues $\&$ volume $=6 \&$ ano $=2010 \& n$ umero $=2$.

\section{Como citar este artigo:}

Scherer EA, Scherer ZAP, Azevedo HA, Durão MAS. Opinião de pacientes psiquiátricos internados sobre o hábito de fumar. SMAD, Rev. Eletrônica Saúde Mental Álcool Drog. (Ed. port.). jan.-abr. 2012 [acesso: 33. Disponível em:

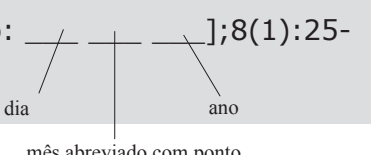

mês abreviado com ponto 